\title{
COMPACT ARRAY MAPPING OF THE
}

\section{NUCLEAR STARBURST IN NGC 7552}

\author{
DUNCAN A. FORBES \\ Lick Observatory, University of California, Santa Cruz, CA 95064, USA \\ RAY P. NORRIS \\ Australia Telescope National Facility, CSIRO, P.O. Box 76, Epping, NSW 2121, \\ Australia \\ and \\ GERRY M. WILLIGER AND R. CHRIS SMITH \\ Cerro Tololo Inter-American Observatory, Casilla 603, La Serena, Chile
}

\begin{abstract}
We discuss new observations of the starburst galaxy NGC 7552. From optical and near-infrared colour maps we find a red, dusty circumnuclear ring. High-resolution radio mapping from the ATCA reveals the same ring, and a number of bright blobs (probably SNRs). The ring is probably associated with gas and dust which have lost angular momenta due to torques in the bar potential and settled at the inner Lindblad resonance. These circumnuclear starburst rings may be relatively common (when mapped without the obscuring affects of dust) and may play a role in collimating material of a nuclear outflow.
\end{abstract}

\section{Observations and Data Reduction}

We observed the starburst galaxy NGC 7552 with the Australia Telescope Compact Array (ATCA) at $4.79 \mathrm{GHz}(6 \mathrm{~cm})$ and $8.64 \mathrm{GHz}(3 \mathrm{~cm})$ simultaneously at the highest resolution available. Our amplitude calibrator (1934-638) was observed at the beginning and the end of the run. The source and the phase calibrator (2311452) were observed continuously for 12 hours. The data were edited and calibrated using the AIPS software package. The final maps were restored with a Gaussian beam. The final 3-cm map has a half power beam width (HPBW) of $1^{\prime \prime}$. The 6-cm map has a HPBW of $2^{\prime \prime}$. At an assumed distance of $32.7 \mathrm{Mpc}, 1^{\prime \prime}$ corresponds to $158 \mathrm{pc}\left(\mathrm{H}_{0}=50 \mathrm{~km} \mathrm{~s}^{-1} \mathrm{Mpc}^{-1}\right)$. Details of the optical and near-infrared imaging will be given elsewhere.

\section{Results}

Optical (B/I) and near-infrared ( $\mathrm{J} / \mathrm{K})$ colour maps reveal a red circumnuclear ring, which presumably contains dust. Several knots or bright blobs are present within the ring. Radio mapping allows us to examine the star formation activity in the nuclear region free from the effects of reddening. NGC 7552 is a nearly face-on ( $i=$ $28^{\circ}$ ) SBbc spiral and therefore an excellent candidate in which to study its nuclear activity. In both the 3-cm and 6-cm maps we observe a weak nucleus surrounded by a ring of emission. The ring emission is not smooth and consists of several bright blobs which, overall, have a steep spectral index, suggesting they are SNRs. Fig. 1 shows the $3-\mathrm{cm}$ map. The ring has dimensions of $1.3 \times 1.1 \mathrm{kpc}$ at position angle $\sim 100^{\circ}$, a width of $\sim 400 \mathrm{pc}$ and $\mathrm{a} / \mathrm{b} \sim 1.2$. It thus corresponds to the ring seen in the colour ratio maps. 


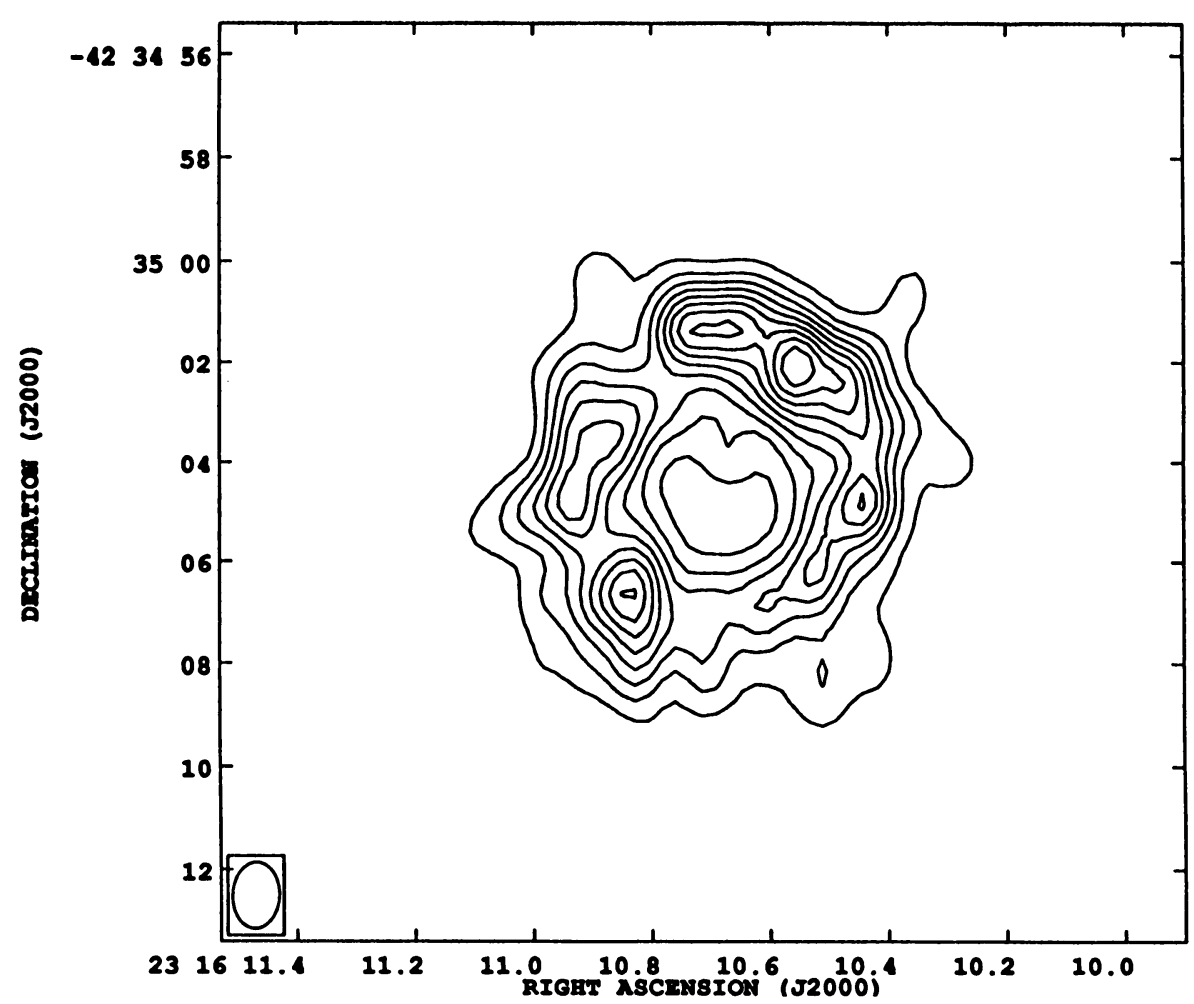

Fig. 1. 3-cm radio map ( 1 " resolution) showing the circumnuclear ring of star formation. Contours are at intervals of $0.27 \mathrm{mJy}$

\section{Discussion}

Perhaps the most interesting feature of NGC 7552 is the circumnuclear ring. Other well-known galaxies (e.g. NGC 613, 1097, \& 1808) also reveal near-circular rings of star formation when deprojected for inclination. Several authors have shown that an interaction, which may lead to the development of a bar potential, can remove angular momentum and kinetic energy from gas clouds, which causes them to sink towards the centre of the galaxy (e.g. Noguchi 1988; Barnes \& Hernquist 1991). Simulations by Noguchi (1988) show the formation of a gas ring at the inner Lindblad resonance (ILR), which coincides with the maximum infall rate of material and the epoch of active star formation. The accumulation and compression of molecular material in a circumnuclear ring may induce a burst of star formation at this location and explain the ring seen in NGC 7552. Although this idea seems attractive, further work is needed to verify it.

Extended [OI] $6300 \AA$ emission in NGC 7552 led Feinstein et al. (1990) to suggest we may be witnessing a face-on 'superwind'. Indeed the $X$-ray to far-infrared ratio $\left(\log \mathrm{L}_{X} / \mathrm{L}_{F I R}\right)$ is -3.74 , which is typical of superwind galaxies (see Heckman, Armus \& Miley 1990). The spectral flux distribution of NGC 7552 shows a 
remarkable similarity to the prototype superwind galaxy M82 over eight orders of frequency (see Feinstein et al. 1990). Rings of dense gas may play a role in collimating such a superwind. In M82, a number of compact radio sources (i.e. SNRs) are located within a region defined by the dense molecular ring. The case of NGC 1808 is particularly interesting. Here we have the compact radio sources (Saikia et al. 1990) and hotspots (Forbes et al. 1992) defined by the inner radius of an HI ring (Koribalski, Dickey \& Mebold 1992). The deprojected region is about $1.6 \mathrm{kpc}$ in diameter (assuming inclination $=57^{\circ}$ and distance $=16.4 \mathrm{Mpc}$ ). Recent evidence for outflowing gas (Koribalski et al. 1992; Phillips 1992) has confirmed its classification as a superwind galaxy. Phillips (1992) finds that the $\mathrm{H} \alpha$ filaments emanate from this region and 'fan out' above the disk.

\section{Acknowledgments}

We thank Rafik Kandalian and Nelson Caldwell for their assistance with the radio and optical observations respectively.

\section{References}

Barnes, J. E., \& Hernquist, L. 1991, ApJ, 370, L65

Feinstein, C., Vega, I., Mendez, M., \& Forte, J. C. 1990, A\&A, 239, 90

Forbes, D. A., Boisson, C., \& Ward, M. J. 1992, submitted to MNRAS

Heckman, T. M., Armus,L., \& Miley, G. K., 1990, ApJS, 74, 833

Koribalski, B., Dickey, J. M., \& Mebold, U. 1992, submitted to ApJL

Koribalski, B., Dahlem, M., Mebold, U., \& Brinks, E. 1992, submitted to A\&A

Noguchi, M. 1988, A\&A, 203, 259

Phillips, A. C. 1992, in preparation

Saikia, D. J., et al. 1990, MNRAS, 245, 397 